\title{
The correlation of fractal structures in the photospheric and the coronal magnetic field
}

\author{
M. Dimitropoulou ${ }^{1}$, M. Georgoulis ${ }^{2}$, H. Isliker ${ }^{3}$, L. Vlahos ${ }^{3}$, A. Anastasiadis ${ }^{4}$, D. Strintzi ${ }^{5}$, and X. Moussas ${ }^{1}$ \\ 1 University of Athens, Department of Physics, 15483 Athens, Greece \\ e-mail: michaila.dimitropoulou@nsn.com \\ 2 Johns Hopkins University Applied Physics Laboratory, 11100 Johns Hopkins Road, Laurel, MD-20723-6099, USA \\ 3 University of Thessaloniki,Department of Physics, 54006 Thessaloniki, Greece \\ 4 Institute for Space Applications and Remote Sensing, National Observatory of Athens, 15236 Penteli, Greece \\ 5 National Technical University of Athens, 15773 Athens, Greece
}

Received 16 February 2009 / Accepted 23 June 2009

\section{ABSTRACT}

\begin{abstract}
Context. This work examines the relation between the fractal properties of the photospheric magnetic patterns and those of the coronal magnetic fields in solar active regions.

Aims. We investigate whether there is any correlation between the fractal dimensions of the photospheric structures and the magnetic discontinuities formed in the corona.

Methods. To investigate the connection between the photospheric and coronal complexity, we used a nonlinear force-free extrapolation method that reconstructs the $3 \mathrm{~d}$ magnetic fields using $2 \mathrm{~d}$ observed vector magnetograms as boundary conditions. We then located the magnetic discontinuities, which are considered as spatial proxies of reconnection-related instabilities. These discontinuities form well-defined volumes, called here unstable volumes. We calculated the fractal dimensions of these unstable volumes and compared them to the fractal dimensions of the boundary vector magnetograms.

Results. Our results show no correlation between the fractal dimensions of the observed $2 \mathrm{~d}$ photospheric structures and the extrapolated unstable volumes in the corona, when nonlinear force-free extrapolation is used. This result is independent of efforts to (1) bring the photospheric magnetic fields closer to a nonlinear force-free equilibrium and (2) omit the lower part of the modeled magnetic field volume that is almost completely filled by unstable volumes. A significant correlation between the fractal dimensions of the photospheric and coronal magnetic features is only observed at the zero level (lower limit) of approximation of a current-free (potential) magnetic field extrapolation.

Conclusions. We conclude that the complicated transition from photospheric non-force-free fields to coronal force-free ones hampers any direct correlation between the fractal dimensions of the $2 \mathrm{~d}$ photospheric patterns and their $3 \mathrm{~d}$ counterparts in the corona at the nonlinear force-free limit, which can be considered as a second level of approximation in this study. Correspondingly, in the zero and first levels of approximation, namely, the potential and linear force-free extrapolation, respectively, we reveal a significant correlation between the fractal dimensions of the photospheric and coronal structures, which can be attributed to the lack of electric currents or to their purely field-aligned orientation.
\end{abstract}

Key words. Sun: corona - Sun: flares - Sun: photosphere

\section{Introduction}

Solar active regions (ARs) have attracted the interest of many researchers over the years, since they are connected with solar energetic events, such as solar flares and CMEs. The relation of AR magnetic complexity with their flare productivity has been lately widely investigated (Meunier 2004; McAteer et al. 2005; Georgoulis 2008). There are many measures of the ARs' complexity, such as their size distribution (Harvey \& Zwaan 1993; Meunier 1999), the length of the main polarity inversion line (Falconer et al. 2006) or the magnetic flux along the polarity inversion line (Schrijver 2007). A common approach nevertheless relies on fractal analysis because the fractal dimension indicates the self-similarity of a structure over several size scales. The sizes of AR magnetic fields are known to display power-law distributions (Harvey \& Zwaan 1993; Abramenko 2005), meaning that the very nature of AR magnetic fields that indicates fractal analysis as a suitable tool for AR complexity determination.

A variety of fractal analysis methods are available nowadays. A basic classification of these methods distinguishes between monofractal and multifractal techniques. An overview of monofractal algorithms has been provided by McAteer et al. (2005), who indicates the perimeter area, the linear size area, and the box counting methods as the most important monofractal methodologies. The pros and cons of these techniques, as well as the resulting discrepancies are discussed in the same work. These techniques have been widely used in the literature: perimeter area technique (Roudier \& Muller 1987; Hirzberger et al. 1997; Meunier 1999; Bovelet \& Wiehr 2001; Janssen et al. 2003), linear area technique (Tarbell et al. 1990; Lawrence 1991; Schrijver et al. 1992; Balke et al. 1993; Meunier 1999), monofractal box-counting technique (Stark et al. 1997; Gallagher et al. 1998; Georgoulis et al. 2002), multifractal boxcounting technique (Lawrence \& Schrijver 1993; Cadavid et al. 1994; Lawrence et al. 1996; Conlon et al. 2008) multifractal wavelet technique: (Hewett et al. 2008). This yields fractal dimensions for the $2 \mathrm{~d}$ photospheric ARs $\left(D_{2 \mathrm{~d}}\right)$ that vary from $\sim 1.1$ up to $\sim 2.0$, depending on the method used and the AR sample under consideration.

The fractal dimension is also useful because it provides a reliable test of various theoretical models and 
simulations against observations. Cellular automata models (Isliker et al. 2000; Vlahos et al. 2002), percolation models (Seiden \& Wentzel 1996; Fragos et al. 2004), random walk diffusion models (Lawrence \& Schrijver 1993), and photospheric magnetoconvection simulations (Janssen et al. 2003) have been tested against observations through their fractal dimension.

The majority of the above-mentioned fractal analyses has been implemented in $2 \mathrm{~d}$ and applied to (mainly photospheric) magnetograms. Solar energetic events, however, take place above the photosphere. Because of the turbulent photospheric motions, the magnetic helicity, and shear generated in the coronal magnetic fields lead to magnetic discontinuities, which can give rise to nanoflares, microflares, flares, and CMEs when some critical threshold is exceeded.

It would therefore be very interesting to seek the fractal dimension $D_{3 \mathrm{~d}}$ describing the complexity of the magnetic field in the $3 \mathrm{~d}$ volume above a given $2 \mathrm{~d}$ magnetogram. Aschwanden and Aschwanden (2008a,b) were among the first to follow this concept. They calculated the $D_{2 \mathrm{~d}}$ fractal dimension of 20 flares, using a standard box-counting technique on data from the Transition Region and Coronal Explorer (TRACE). To infer the $D_{3 \mathrm{~d}}$ in the corona, they created an analytical flare geometry model. The coronal arcade geometry includes three free parameters (arcade length, width, and heliographic longitude), and makes a number of simplifying assumptions, such as (1) the arcade is near the equator and latitudinal projection effects are neglected; (2) the magnetic shear along the neutral line is neglected; (3) the neutral line is oriented in the east-west direction; (4) individual loops in the arcade are semicircular; and (5) the arcade length is assumed to be commensurable with the arcade width when observed near the limb. This work has shown that $D_{2 \mathrm{~d}}$ and $D_{3 \mathrm{~d}}$ do not scale as $D_{2 \mathrm{~d}} / 2=D_{3 \mathrm{~d}} / 3$, as expected for isotropic structures, but obey a more complex relation.

The purpose of the present work is to elaborate the relation between $D_{2 \mathrm{~d}}$ and $D_{3 \mathrm{~d}}$ further by using - for the first time - magnetic field measurements and extrapolations. We investigate whether a correlation between $D_{2 \mathrm{~d}}$ and $D_{3 \mathrm{~d}}$ actually exists and we have created an extended database of 38 ARs in the form of vector magnetograms. Our input magnetograms come both from the Imaging Vector Magnetograph (IVM; Mickey et al. 1996; LaBonte et al. 1999) of the Mees Solar Observatory and the spectropolarimeter (SP; Lites et al. 2001) of the solar optical telescope (SOT) onboard Hinode. We first calculated the $D_{2 \mathrm{~d}}$ of the $2 \mathrm{~d}$ magnetic structures captured by the magnetograms through a conventional box-counting algorithm. To infer the $D_{3 \mathrm{~d}}$ of the volume above a given AR, we extrapolated the observed photospheric magnetograms into the corona. The extrapolation method-of-choice was the nonlinear force-free optimization method of Wiegelmann (2004), extending the work of Wheatland et al. (2000). Although nonlinear force-free extrapolation is still a fully open research topic, Wiegelmann's (2004) method has been recognized by comparison studies involving several nonlinear force-free extrapolation methods as one of the most reliable techniques (e.g. Schrijver et al. 2006; Metcalf et al. 2008). Potential extrapolation was also used as a zero level of approximation for reasons of comparison. After the extrapolation, we identified unstable volumes (UnVos) in the corona as ensembles of adjacent magnetic discontinuity sites. The $3 \mathrm{~d}$ fractal dimension $D_{3 \mathrm{~d}}$ is determined as the box-counting fractal dimension of these UnVos.

This work is structured as follows. Section 2 describes the data used in this study along with the necessary corrections imposed on them. Section 3 discusses the box counting and extrapolation techniques applied to our dataset, aiming to reveal correlations between $2 \mathrm{~d}$ and $3 \mathrm{~d}$ magnetic domains. Important parameters and quantities used are explained in detail, whereas work-around solutions on standard methodology drawbacks are suggested. Section 4 presents our results and discusses our findings. Finally, Sect. 5 summarizes our conclusions.

\section{Dataset}

Nonlinear force-free extrapolation techniques typically require vector magnetograms that are not as widely available as conventional line-of-sight magnetograms. Here we have created a database of 38 different AR vector magnetograms from the IVM and Hinode's SOT/SP.

The IVM obtains Stokes images in photospheric lines with $7 \mathrm{pm}$ spectral resolution, 1 arcsec spatial resolution $(\sim 0.55$ arcsec per pixel in full resolution) over a field of $4.7 \mathrm{arcmin}^{2}$ and polarimetric precision of $0.1 \%$ (Mickey et al. 1996). We used both fully-inverted and quicklook IVM data. The quicklook data were obtained from the IVM Survey Data archive, made available at http://www. cora.nwra.com/ ivm/IVM-SurveyData/. The quicklook data reduction differs from the complete inversion in that it uses a simplified flat-fielding approach, takes no account of scattered or parasitic light, and no correction is attempted for seeing variations that occur during the data acquisition. On the other hand, the spectro polarimeter (SP) of SOT onboard Hinode obtains line profiles of two magnetically sensitive Fe lines at 630.15 and $630.25 \mathrm{~nm}$, using a $0.16 \times 164$ arcsec slit and has a spectral resolution of $30 \mathrm{~mA}$ (Shimizu 2004).

In this study we used 10 fully inverted and 16 quicklook IVM vector magnetograms with 12 Level1D SOT magnetograms. To remove the intrinsic azimuthal ambiguity of $180^{\circ}$ we used the Non-Potential magnetic Field Calculation (NPFC) method of Georgoulis (2005). For computational convenience we also rebinned the disambiguated magnetograms into a $128 \times 128$ grid.

That our input data derive from instruments with different spatial resolutions should not influence our analysis, as the fractal dimension is scale-invariant and does not depend on the spatial resolution of the instrument used. This is true for mathematical fractals however, because fractality holds for a finite range of sizes in realistic situations. For this reason, we first assume that all multi-instrument vector magnetograms belong to the same dataset, and then we duplicate the analysis using three different sets: one including fully-inverted IVM magnetograms, one including quicklook IVM data, and one including the Hinode/SOT data.

\section{Data analysis method}

Our analysis consists of 3 distinct steps. First we apply a boxcounting algorithm to the $128 \times 128$ disambiguated photospheric magnetograms. We estimate the $D_{2 \mathrm{~d}}$ fractal dimension from the pixels with magnetic field strength exceeding a given threshold. Second, we apply the Wiegelmann optimization algorithm to our vector magnetograms in order to nonlinearly extrapolate the magnetic field from the photospheric boundary. We thus construct a $3 \mathrm{~d} 128 \times 128 \times 128$ cube, within which the magnetic field is unambiguously determined. We also perform potential extrapolation as a zero level of approximation. Third, we calculate $D_{3 \mathrm{~d}}$ via box-counting the sites within our cubic grid which exceed a threshold of either the gradient of the magnetic field strength or the magnitude of the rotation (curl) of the magnetic field vector. The UnVos in the coronal volume are determined in this way. Finally, we investigate whether there is any correlation between 
the inferred $D_{2 \mathrm{~d}}$ and $D_{3 \mathrm{~d}}$. The quality of the shown correlations is being judged by both the linear (Pearson) correlation coefficients.

\section{1. $D_{2 \mathrm{~d}}$ Determination}

To calculate the $D_{2 \mathrm{~d}}$ of each $\mathrm{AR}$ on the photospheric level, we apply a standard box-counting technique, similar to McAteer et al. (2005). As input data we use the disambiguated $128 \times 128$ vector magnetograms (see Sect. 2). The quantity examined is the magnetic field magnitude $|\boldsymbol{B}|$ at the photospheric level $(z=0)$, which can be unambiguously determined by the known magnetic field components $\boldsymbol{B}_{\boldsymbol{x}}, \boldsymbol{B}_{\boldsymbol{y}}, \boldsymbol{B}_{z}$ on the heliographic plane. A site is taken into account in the box-counting if its magnetic field magnitude exceeds a specific threshold $B_{\mathrm{cr}}$. If the number, $N$, of strongly magnetized square boxes $\left(|\boldsymbol{B}| \geq B_{\mathrm{cr}}\right)$ scales with box size, $\epsilon$, as

$N(\epsilon) \propto \epsilon^{-D_{2 \mathrm{~d}}}$

then $D_{2 \mathrm{~d}}$ is the fractal dimension, whereby $\epsilon$ is successively increased as $\epsilon=2^{n}, n=0,1,2, \ldots 7$, with maximum $\epsilon_{\max }=128$. In practice, $D_{2 \mathrm{~d}}$ is the scaling index of the power-law least-squares best fit between $N(\epsilon)$ and $\epsilon$. The criterion $|\boldsymbol{B}| \geq B_{\text {cr }}$ for identifying the photospheric ARs has been used widely in the literature (e.g. McAteer et al. 2005).

However, the above-mentioned methodology is known to suffer from two major problems. First and foremost, it is sensitive to the selection of the critical threshold value $B_{\mathrm{cr}}$. To determine a non-arbitrary value of $B_{\mathrm{cr}}$, we apply a histogram method, by constructing the histogram of the field magnitudes of all magnetograms in our database. We then fit a Gaussian to this histogram and define $B_{\mathrm{cr}}$ as the value, above which the histogram deviates from the Gaussian. This test yields $B_{\mathrm{cr}}=230 \mathrm{G}$. The second drawback lies in the least-squares best fit being prone to large errors, mainly due to the small number of data-points in its dynamical range. To overcome this problem, we apply a goodness of fit test in the form of a chi-square test, following Isliker (1992). The chi-square test is applied to a sliding window on the linear representation of the scaling relation, and it indicates with a $90 \%$ level of significance whether a range of the overall scaling is indeed a power law. In addition, we demand that the powerlaw scaling extends over at least one order of magnitude, in order to yield sufficient dynamical range for a reliable estimate of the fractal dimension. As an example in Fig. 1 we show the plot of $\log N_{\epsilon}$ versus $\log \epsilon$ for AR 10953, where $D_{2 \mathrm{~d}}=1.63$.

\subsection{Nonlinear force-free extrapolation}

The next step is to extrapolate the photospheric magnetic fields. Potential extrapolation is the lower limit (zero level of) approximation and it provides the simplest force-free magnetic configuration. As such, it is expected to yield the best correlation between $D_{2 \mathrm{~d}}$ and $D_{3 \mathrm{~d}}$. A linear, but non-potential, force-free field extrapolation is the first level of approximation, while the nonlinear force-free (NLFF) field extrapolation is the second level of approximation. The most realistic treatment would be a non-force-free static extrapolation or a magnetohydrostatic/magnetohydrodynamic model using the photospheric fields as boundary conditions. The latter, however, implies analysis and computational resources that far exceed the scope of this work. We chose the NLFF field extrapolation for two reasons:

1. while the linear force-free assumption may have some validity in a minimum-energy AR corona, it cannot be trusted

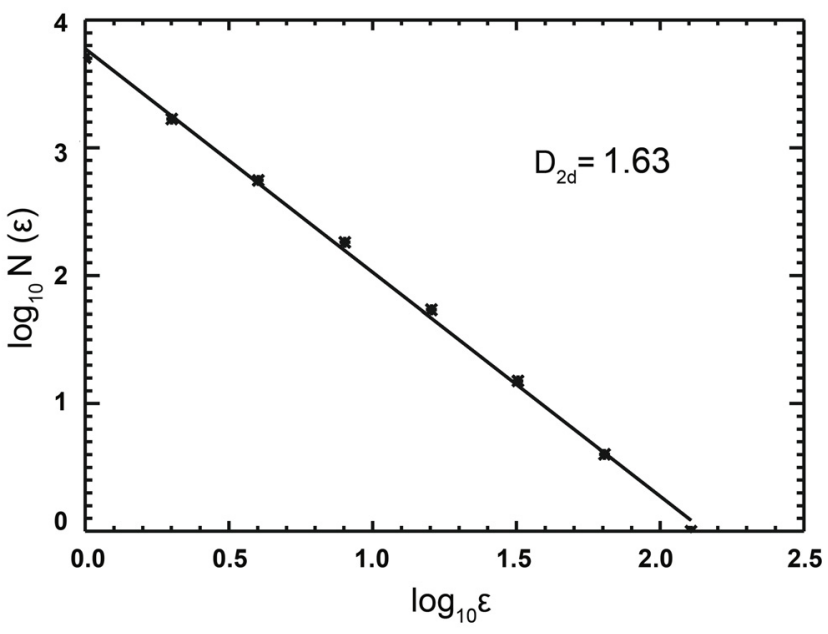

Fig. 1. Logarithmic plot of $N(\epsilon)$ versus $\epsilon$ for AR 10953, where $D_{2 \mathrm{~d}}=$ 1.63.

in the chromosphere or, even more, in the AR photosphere. There the relatively high value of the plasma beta-parameter implies Lorentz forces that cannot be neglected;

2 . because the purpose of this work is to investigate whether a correlation between $D_{2 \mathrm{~d}}$ and $D_{3 \mathrm{~d}}$ exists, it is crucial to refrain from using an extrapolation that is susceptible to artificial correlations between the $2 \mathrm{~d}$ and the $3 \mathrm{~d}$ domains. A linear extrapolation method would bind the $3 \mathrm{~d}$ coronal magnetic field to its photospheric $2 \mathrm{~d}$ boundary.

The NLFF extrapolation used here is based on the optimization technique introduced by Wheatland et al. (2000) and further developed by Wiegelmann (Wiegelmann 2004; Wiegelmann et al. 2006; Wiegelmann 2008). This technique reconstructs force-free magnetic fields from their boundary values, based on minimizing the Lorentz force and the divergence of the magnetic field vector in the extrapolation volume:

$L=\int_{V} w(x, y, z)\left[|\boldsymbol{B}|^{-2}|(\boldsymbol{\nabla} \times \boldsymbol{B}) \times \boldsymbol{B}|^{2}+|\nabla \cdot \boldsymbol{B}|^{2}\right] \mathrm{d}^{3} x$.

In the above functional, $w(x, y, z)$ is a weighting function and $V$ denotes the extrapolation volume. A force-free state is reached when $L=0, w>0$. For $w(x, y, z)=1$, the magnetic field must be available on all 6 boundaries of our cubic box for the optimization algorithm to work. Nevertheless, real vector magnetograms provide the magnetic field only for the bottom boundary, whereas the edge top and lateral magnetic field values remain unknown. The weighting function is thus used to reduce the dependence of the interior solution on the unknown boundaries. We introduced a buffer boundary of 100 grid points towards the lateral and top boundaries of the computational box. We then chose $w(x, y, z)=1$ in the inner, physical domain and let $w$ drop to 0 with a cosine-profile in the buffer boundary towards the lateral and top boundaries of the computational box (see Wiegelmann 2004, for details).

An additional useful attribute of Wiegelmann's NLFF field extrapolation code is the preprocessing alternative it offers. As the photospheric magnetic field is in principle inconsistent with the force-free approximation, a preprocessing procedure was developed by Wiegelmann et al. (2006) in order to drive NLFF fields closer to a force-free equilibrium. Preprocessing minimizes the forces and torques in the system thus satisfying the 


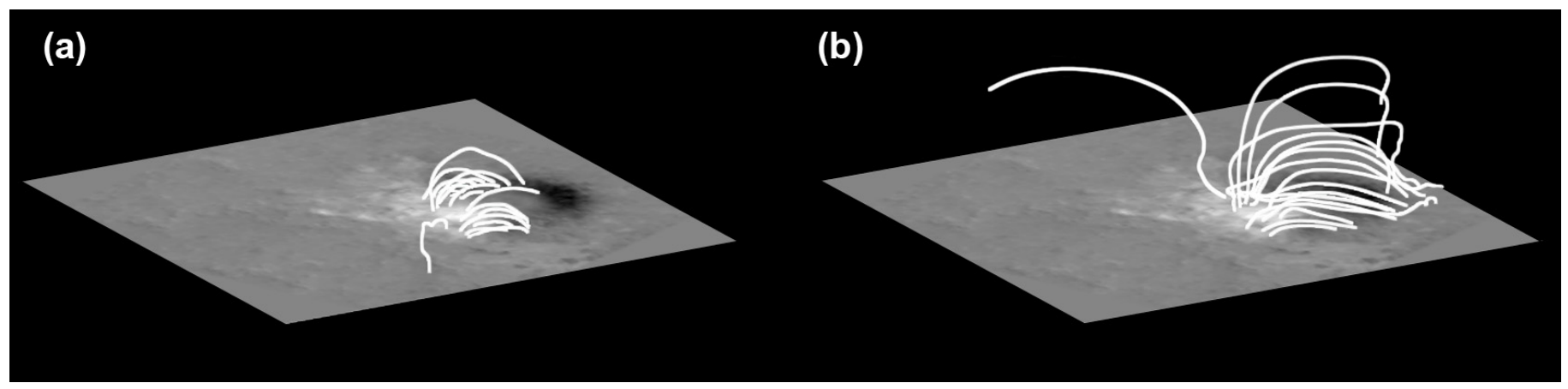

Fig. 2. a) Potential extrapolation for AR 10953. b) The respective NLFF field solution, where the boundary vector magnetogram has been preprocessed.

force-free requirements more closely. While preprocessing the photospheric magnetograms, however, we applied some smoothing to the field components. To study the effects of this smoothing we duplicated the fractal-dimension calculation for unpreprocessed photospheric fields, as well. As shown in Sect. 4, preprocessing - including smoothing - does not affect $D_{2 \mathrm{~d}}$ significantly.

Figure 2 depicts the field lines of the extrapolated magnetic field for AR 10953, as calculated by the optimization algorithm. Frame a) shows the current-free (potential) extrapolation for reference while the respective NLFF field solution is shown in Frame b).

\section{3. $D_{3 \mathrm{~d}}$ determination}

The fractal dimension $D_{3 \mathrm{~d}}$ in the extrapolation volume is determined by means of the box-counting and goodness-of-fit methods described in Sect. 3.1. What differs in this case is the quantity used to determine which grid sites in the volume will be taken into account. This quantity will determine the UnVos present in the cubic grid. We have two different ways of calculating these UnVos:

1. The average magnetic field gradient $G_{\mathrm{av}}$.

For every site $i, j, k$ within our grid, we calculate the average magnetic field gradient with its neighboring sites as

$$
\begin{aligned}
& G_{\mathrm{av}_{i, j, k}}=\frac{\left|\boldsymbol{B}_{i, j, k}-\boldsymbol{B}_{\text {sum }} / n n\right|}{\left|B_{i, j, k}\right|} \\
& \boldsymbol{B}_{\text {sum }}=\left(B_{\text {sum } x}, B_{\text {sum } y}, B_{\text {sumz } z}\right) .
\end{aligned}
$$

We define:

$B_{\text {sum } x}=B_{x_{i+1, j, k}}+B_{x_{i-1, j, k}}+B_{x_{i, j+1, k}}+B_{x_{i, j-1, k}}+B_{x_{i, j, k+1}}+B_{x_{i, j, k-1}}$

$B_{\text {sumy } y}=B_{y_{i+1, j, k}}+B_{y_{i-1, j, k}}+B_{y_{i, j+1, k}}+B_{y_{i, j-1, k}}+B_{y_{i, j, k+1}}+B_{y_{i, j, j-1}}$

$B_{\text {sumz }}=B_{z_{i+1, j, k}}+B_{z_{i-1, j, k}}+B_{z_{i, j+1, k}}+B_{z_{i, j-1, k}}+B_{z_{i, j, k+1}}+B_{z_{i, j, k-1}}$.

Depending on the location of each site within the volume, the number of nearest neighbors $n n$ assumes the values $n n=$ $3,4,5,6$. The physical explanation for selecting this criterion lies in a steep gradient of the magnetic field strength being thought to favor magnetic reconnection in $3 \mathrm{~d}$, in the absence of null points (Priest et al. 2003).

2. The normalized magnetic field curl $C_{n}$.

For every site $i, j, k$ within our grid we calculate the normalized magnetic field curl as $C_{n_{i, j, k}}=\left|\frac{\nabla \times B_{i, j, k}}{B_{i, j, k}}\right|$.

This criterion will obviously highlight areas of high electriccurrent concentrations, which are known to play a role in the formation of magnetic instabilities.
During the application of the box-counting method, we consider a site as unstable if it exceeds a critical threshold value (gradient or curl respectively). This critical value is determined with the histogram method described in Sect. 3.1, yielding $G_{\mathrm{cr}}=0.09$ for the average gradient criterion and $C_{\mathrm{cr}}=0.005$ for the normalized curl one. If the number, $N$, of unstable square boxes $\left(G_{\mathrm{av}} \geq G_{\mathrm{cr}}\right.$ or $C_{n} \geq C_{\text {cr }}$ ) scales with box size, $\epsilon$, as

$N(\epsilon) \propto \epsilon^{-D_{3 \mathrm{~d}}}$

then $D_{3 \mathrm{~d}}$ is the $3 \mathrm{~d}$ fractal dimension, whereby $\epsilon$ is once again successively increased as $\epsilon=2^{n}, n=0,1,2, \ldots 7$, with maximum $\epsilon_{\max }=128$. As an example in Fig. 3 we show the logarithmic plot of $N(\epsilon)$ versus $\epsilon$ for AR 10953, where $D_{3 \mathrm{~d}}=2.52$ for the gradient (frame a) and $D_{3 \mathrm{~d}}=2.91$ for the curl (frame b). In both cases preprocessing were conducted prior to the NLFF extrapolation.

\section{Results}

As discussed in Sect. 3.2, several combinations were investigated with respect to the extrapolation methods and correlation coefficients. We first present the case of NLFF extrapolation with preprocessing. This is carried out before NLFF extrapolation, as unpreprocessed magnetograms are not consistent with force-free extrapolations. Consequently it would be very hard to approximate a nonlinear force-free equilibrium consistent with unpreprocessed data.

First we investigate the relation between $D_{2 \mathrm{~d}}$ and $D_{3 \mathrm{~d}}$, when the latter is calculated through the average $\left(G_{\mathrm{av}}\right)$ magnetic field gradient. The corresponding Pearson correlation coefficient between $D_{2 \mathrm{~d}}$ and $D_{3 \mathrm{~d}}$ is -0.154 . The corresponding probabilities are shown in Table 1 for Pearson coefficient. We then use the normalized magnetic field curl $\left(C_{n}\right)$, to calculate $D_{3 \mathrm{~d}}$. In this second attempt the Pearson correlation coefficient between $D_{2 \mathrm{~d}}$ and $D_{3 \mathrm{~d}}$ is 0.251 , as shown along with the corresponding probabilities in Table 1. Evidently, when NLFF extrapolation is used, there is no correlation between $D_{2 \mathrm{~d}}$ and $D_{3 \mathrm{~d}}$ at the $95 \%$ significance level and this result is independent of the criterion used to quantify the UnVos. Moreover, independently of the magnetic complexity of an AR in the photosphere (as quantified by $D_{2 \mathrm{~d}}$ ), the complexity of the generated UnVos varies within a more or less well-defined range, as can be seen in Table 2. The absence of correlation between $D_{2 \mathrm{~d}}$ and $D_{3 \mathrm{~d}}$, as well as the range of $D_{3 \mathrm{~d}}$ values, is shown in frame a) of Fig. 4, where the NLFF case is presented. This figure suggests that - although the complexity in the photosphere may vary significantly - the UnVos in the corona retain a more or less well-defined behavior.

If we attempt to locate these UnVos within the $128 \times$ $128 \times 128$ volume, one finds that more than $80 \%$ of them are 
(a)

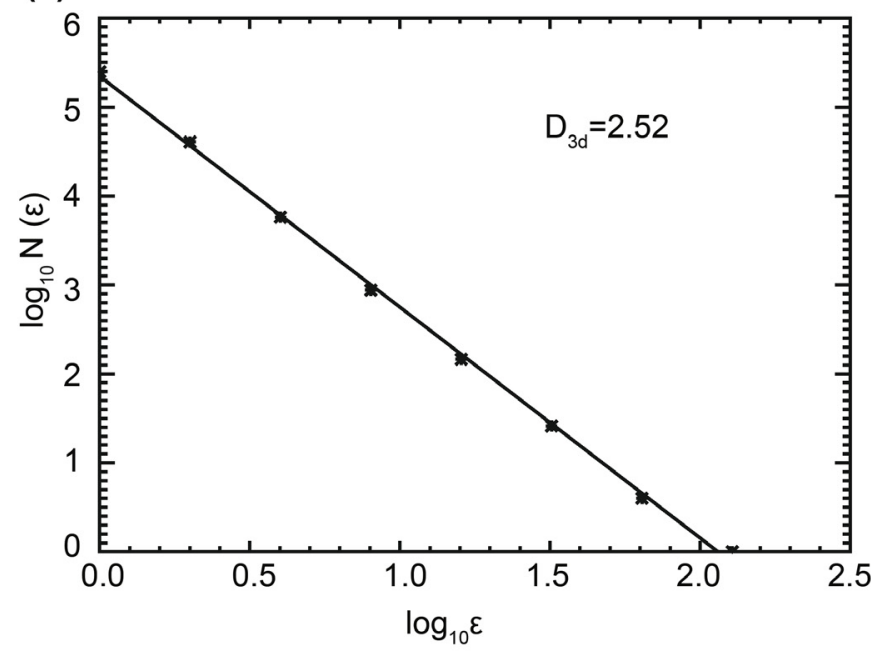

(b)

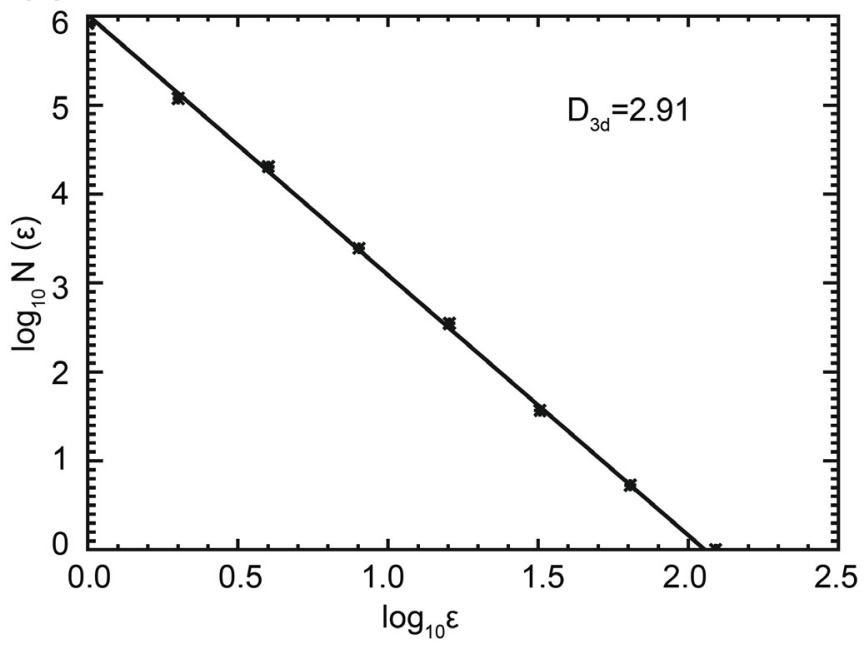

Fig. 3. Logarithmic plot of $N(\epsilon)$ versus $\epsilon$ for the UnVos in AR 10953. UnVos have been calculated using $G_{\text {av }}$ (Frame a) and $C_{n}$ (Frame b). The respective fractal dimensions $D_{3 \mathrm{~d}}$ are also shown.

(a)

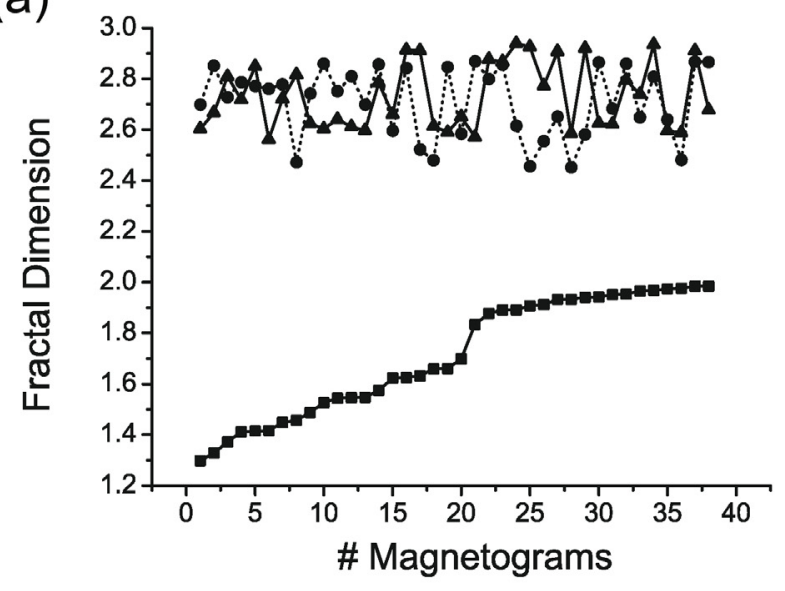

(b)

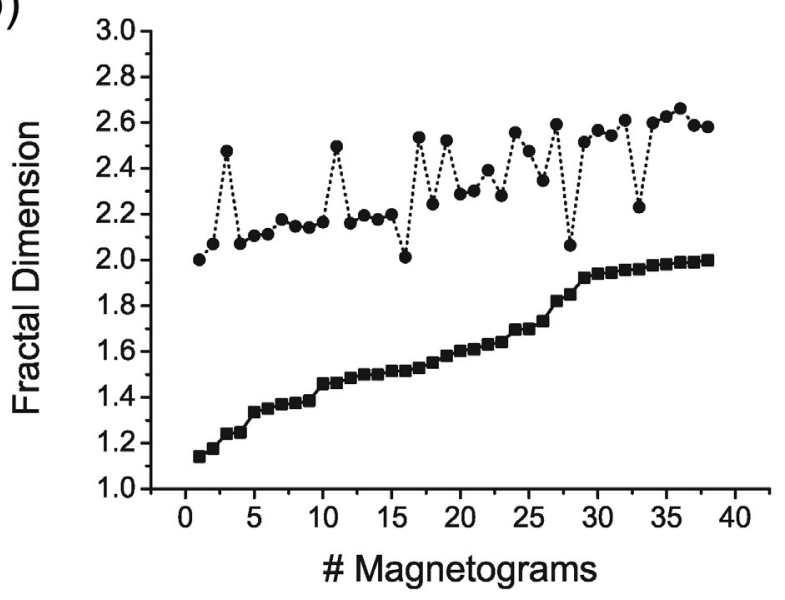

Fig. 4. Fractal dimensions $D_{2 \mathrm{~d}}$ and $D_{3 \mathrm{~d}}$ for all 38 magnetograms in our sample. Frame a) depicts the case of NLFF extrapolation with preprocessing, whereas frame b) depicts the case of potential extrapolation. In both Frames a) and b) the solid line with squares represents $D_{2 \mathrm{~d}}$ in ascending order and the dotted line with circles stands for $D_{3 \mathrm{~d}}$ when UnVos are identified by the $G_{\mathrm{av}}$ criterion, In Frame a) the solid line with triangles reflects $D_{3 \mathrm{~d}}$ when UnVos are identified based on the $C_{n}$ criterion.

Table 1. Pearson correlation coefficients between $D_{2 \mathrm{~d}}$ and $D_{3 \mathrm{~d}}$ for the potential and the NLFF cases.

\begin{tabular}{cccc}
\hline \hline Extrapolation & $\begin{array}{c}\text { Used 3d } \\
\text { criterion }\end{array}$ & $\begin{array}{c}\text { Correlation } \\
\text { with } D_{2 \mathrm{~d}}\end{array}$ & $\begin{array}{c}\text { Corresponding } \\
\text { Probability }\end{array}$ \\
\hline Pothod & $G_{\mathrm{av}}$ & 0.719 & $99 \%$ \\
NLFF with preprocessing & $G_{\mathrm{av}}$ & -0.154 & $65 \%$ \\
NLFF with preprocessing & $C_{n}$ & 0.251 & $87 \%$ \\
\hline
\end{tabular}

accumulated in the lower layers, whereas only $20 \%$ are found at heights $15 \leq z \leq 30$, in units of the boundary magnetogram's linear pixel size. The magnetic discontinuities are restricted to the lower coronal layers, whereas only a few weak additional discontinuities are identified in higher $z \geq 30$ layers. This result is even better illustrated if we slice our volume in layers along the $z$ axis and mark which sites per layer host magnetic instabilities. Figure 5 shows the results for AR 10953, where $G_{\mathrm{av}}$ is used to
Table 2. Range of $D_{2 \mathrm{~d}}$ and $D_{3 \mathrm{~d}}$ values, depending on the criterion used. $100 \%$ of UnVos are considered.

\begin{tabular}{cccc}
\hline \hline $\begin{array}{c}\text { Extrapolation } \\
\text { Method }\end{array}$ & $\begin{array}{c}\text { Fractal } \\
\text { Dimension }\end{array}$ & $\begin{array}{c}\text { Used } \\
\text { criterion }\end{array}$ & $\begin{array}{c}\text { Range of } \\
\text { values }\end{array}$ \\
\hline Potential & $D_{2 \mathrm{~d}}$ & $B_{\mathrm{cr}}$ & $1.57 \pm 0.43$ \\
Potential & $D_{3 \mathrm{~d}}$ & $G_{\mathrm{av}}$ & $2.331 \pm 0.331$ \\
NLFF with preprocessing & $D_{2 \mathrm{~d}}$ & $B_{\mathrm{cr}}$ & $1.64 \pm 0.34$ \\
NLFF with preprocessing & $D_{3 \mathrm{~d}}$ & $G_{\mathrm{av}}$ & $2.66 \pm 0.21$ \\
NLFF with preprocessing & $D_{3 \mathrm{~d}}$ & $C_{n}$ & $2.75 \pm 0.19$ \\
\hline
\end{tabular}

determine UnVos in heights $z=17,22,27,32$ correspondingly. It is obvious that the higher they are from the photosphere, the less and the weaker they are. This can be shown alternatively by means of the fractal dimension: we identify which sites per layer $z_{o}$ satisfy the relation $G_{\mathrm{av}_{i, j, z_{o}}} \geq G_{\mathrm{cr}}$ and by using the box counting technique we calculate the fractal dimension $D_{2 \mathrm{~d}_{z o}^{\prime}}^{\prime}$. The 
(a)

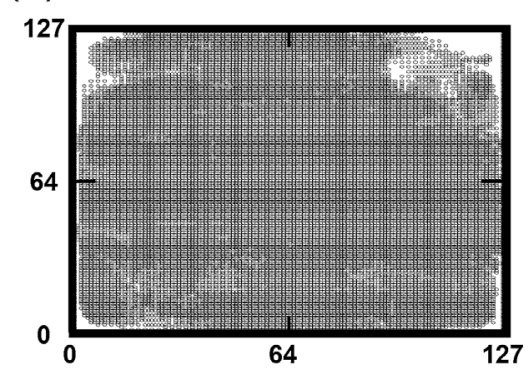

(c)

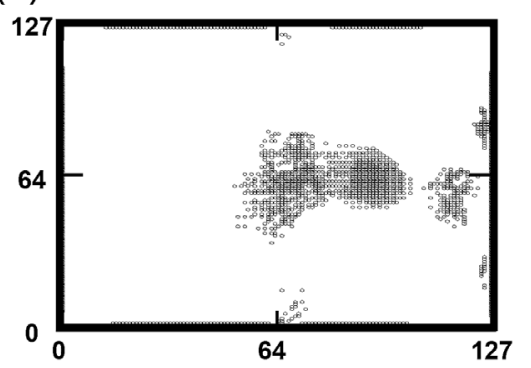

(b)

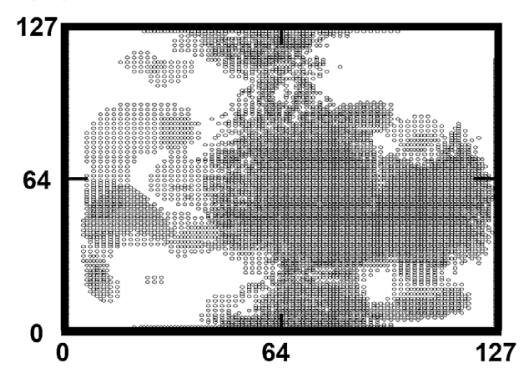

(d)

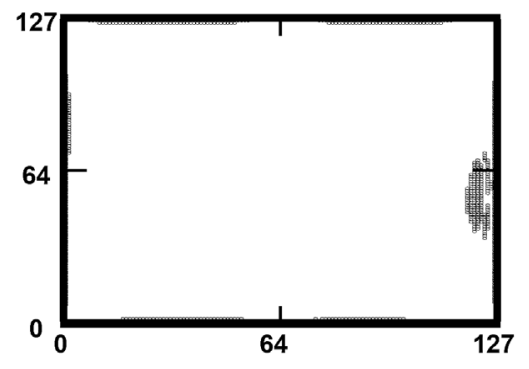

Fig. 5. Sites per $z$ layer that host magnetic instabilities according to the $G_{\mathrm{av}}$ criterion for AR 10953. a) $z=17$, b) $z=22$, c) $z=27$, d) $z=32$.

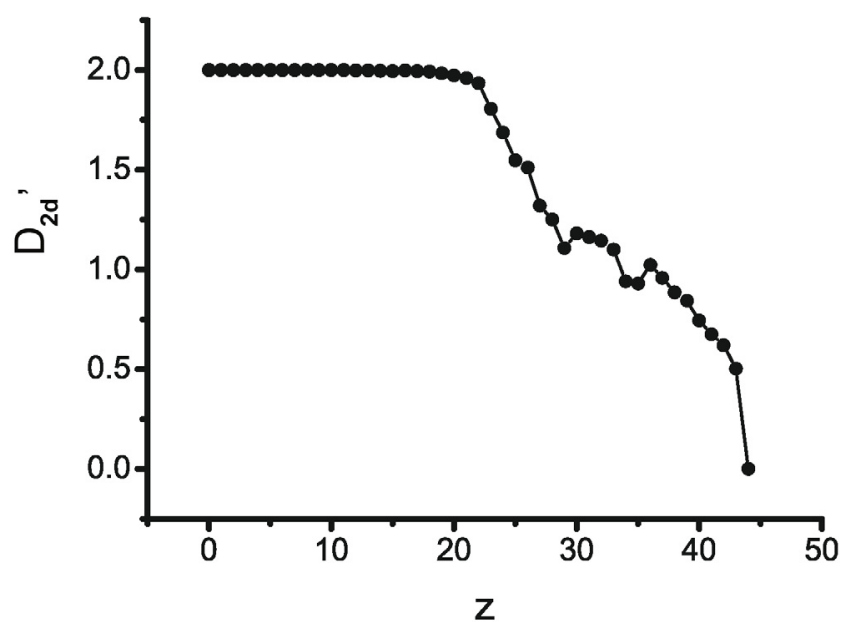

Fig. 6. Fractal dimension $D_{2 \mathrm{~d}}^{\prime}$ versus height $z$ for AR 10953 .

distribution of $D_{2 \mathrm{~d}}^{\prime}$ with height is shown in Fig. 6. It is evident that each layer for $0 \leq z \leq 20$ is almost completely filled by UnVos, yielding a nearly Euclidian (non-fractal) dimension of $D_{2 \mathrm{~d}}^{\prime} \simeq 2$. For heights $20 \leq z \leq 30, D_{2 \mathrm{~d}}^{\prime}$ decreases from 2 to 1 , while for $z>30 D_{2 \mathrm{~d}}^{\prime}$ becomes smaller than 1 , indicating very small and isolated, "dust-like", UnVos.

This finding corroborates previous results. Regnier \& Priest (2007) show that there is a clear preference for free energy accumulation very close to the photospheric level. Given this physical property, we attempted an alternative approach by omitting the layers that are filled by UnVos and focusing only on the part of the volume where the filling with UnVos starts becoming sparse. In this case, would there be any correlation between the photospheric driver and the higher coronal structures? To examine this possibility, we calculated $D_{3 \mathrm{~d}}$ starting from the specific $z$, above which only the remaining $20 \%$ of UnVos is identified, thus excluding the lower coronal layers with a large filling factor. This leads to a decrease in the fractal dimension $D_{3 \mathrm{~d}}$, as expected, and an increase in the correlation coefficients, but we still find a lack of any significant correlation between $D_{2 \mathrm{~d}}$ and $D_{3 \mathrm{~d}}$.

As the first row of Table 1 shows, the only case where we see a considerable correlation at the level of $95 \%$ significance between $D_{2 \mathrm{~d}}$ and $D_{3 \mathrm{~d}}$ is when we use potential field extrapolation. Figure $4 \mathrm{~b}$ illustrates the correlation between $D_{2 \mathrm{~d}}$ and $D_{3 \mathrm{~d}}$ when the magnetic gradient criterion is used in the potential case. This is a reasonable result, considering that the potential extrapolation produces the simplest force-free magnetic configuration. At this zero level of approximation the significant correlation revealed between the photospheric and coronal structures is attributed to the lack of currents. Similar results can also be reproduced for the linear force-free extrapolation: the correlation between $D_{2 \mathrm{~d}}$ and $D_{3 \mathrm{~d}}$ is again significant at the level of $99 \%$, with a Pearson correlation coefficient of 0.75. Inspecting Fig. 4 and Table 2, we also notice that $D_{3 \mathrm{~d}}$ is larger for NLFF field extrapolations compared to potential field extrapolations. This means more extended UnVos, hence a higher spatial filling in the NLFF case, as expected. Other than that, UnVos show a strong preference for accumulating at low altitudes in the extrapolation volume for both NLFF and potential fields. Similar results were found by Vlahos \& Georgoulis (2004), where linear force-free extrapolation is used. Their study does not apply a fractal analysis but identifies many magnetic discontinuities whose free magnetic energies and volumes obey well-formed power-law distribution functions.

Furthermore, we investigated whether the smoothing imposed by the preprocessing has severely affected the magnetic field. For this purpose we compared the fractal dimensions $D_{2 \mathrm{~d}}$ of the observed (unpreprocessed) and the preprocessed magnetograms in the photospheric boundary. As shown in Table 3, the fractal dimension in the photospheric level is not significantly altered by preprocessing. The correlation between the preprocessed and raw magnetic data is significant at a level of $99 \%$, as also illustrated in Fig. 7. While no evidence of correlation between $D_{2 \mathrm{~d}}$ and $D_{3 \mathrm{~d}}$ exists in the NLFF field limit, significant correlation exists in the potential limit, even without preprocessing. 


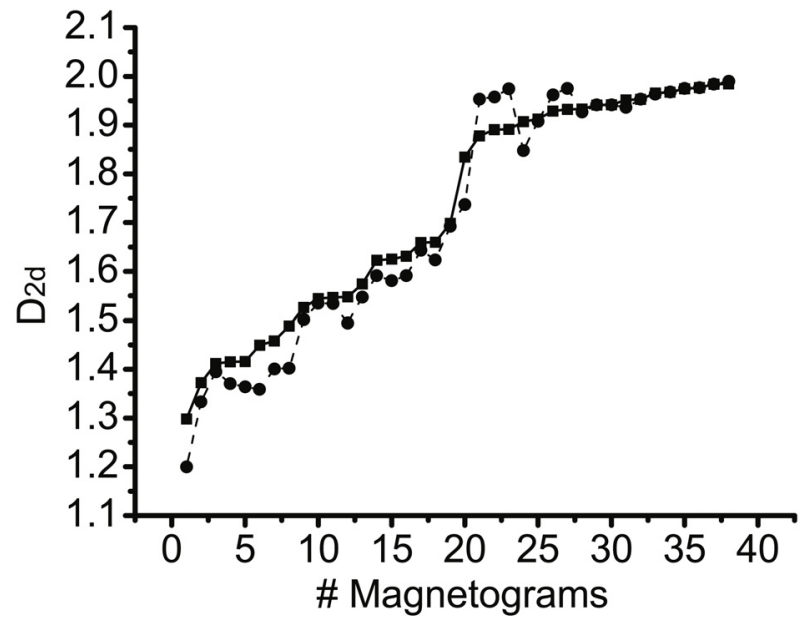

Fig. 7. Fractal dimensions $D_{2 \mathrm{~d}}$ for raw (dashed line with circles) and preprocessed (straight line with squares) magnetograms.

Table 3. Pearson correlation coefficient of the fractal dimensions $D_{2 \mathrm{~d}}$ between the unpreprocessed and the preprocessed photospheric magnetograms.

\begin{tabular}{ccc}
\hline \hline & $\begin{array}{c}\text { Correlation } \\
\text { Coefficient }\end{array}$ & $\begin{array}{c}\text { Corresponding } \\
\text { Probability }\end{array}$ \\
\hline Pearson & 0.863 & $99 \%$ \\
\hline
\end{tabular}

Table 4. Fully Inverted IVM subset: Pearson correlation coefficients between $D_{2 \mathrm{~d}}$ and $D_{3 \mathrm{~d}}$ for the potential and the NLFF cases.

\begin{tabular}{cccc}
\hline \hline Extrapolation & $\begin{array}{c}\text { Used 3d } \\
\text { criterion }\end{array}$ & $\begin{array}{c}\text { Correlation } \\
\text { with } D_{2 \mathrm{~d}}\end{array}$ & $\begin{array}{c}\text { Corresponding } \\
\text { Probability }\end{array}$ \\
\hline Potential & $G_{\mathrm{av}}$ & 0.758 & $99 \%$ \\
NLFF with preprocessing & $G_{\mathrm{av}}$ & -0.278 & $61 \%$ \\
NLFF with preprocessing & $C_{n}$ & -0.102 & $25 \%$ \\
\hline
\end{tabular}

Finally, we investigated how the spatial resolution differences between the datasets derived from various instruments influence our results. We separated the calculated fractal dimensions $\left(D_{2 \mathrm{~d}}\right.$ and $\left.D_{3 \mathrm{~d}}\right)$ into three groups, depending on the instrument they are derived from. This separation substantially decreases the number of data in each sample and is prompt to influence our statistical results. Nevertheless, this is the only way to investigate how different spatial resolution and inversion sophistication (in case of IVM data) can affect the analysis. Poor statistics will lead to relatively low confidence levels. To properly define the threshold values for each of the subsets, we applied the histogram test described in Sects. 3.1 and 3.2 for $2 \mathrm{~d}$ and $3 \mathrm{~d}$ correspondingly separately to each subset; nevertheless, the derived threshold values in all cases were very close to the global $B_{\mathrm{cr}}, G_{\mathrm{cr}}$, and $C_{\mathrm{cr}}$ values found for all 38 magnetograms, thus allowing us to retain them also for all separate subsets.

Table 4 corresponds to Table 1 but only contains the fully inverted data derived from IVM (10 magnetograms). Table 5 corresponds to Table 1 but only contains the data derived from HINODE (12 magnetograms). Table 6 corresponds to Table 1 but only contains the quicklook data derived by IVM (16 magnetograms). Finally, Table 7 corresponds to Table 3 but for each
Table 5. HINODE subset: Pearson correlation coefficients between $D_{2 \mathrm{~d}}$ and $D_{3 \mathrm{~d}}$ for the potential and the NLFF cases.

\begin{tabular}{cccc}
\hline \hline $\begin{array}{c}\text { Extrapolation } \\
\text { Method }\end{array}$ & $\begin{array}{c}\text { Used 3d } \\
\text { criterion }\end{array}$ & $\begin{array}{c}\text { Correlation } \\
\text { with } D_{2 \mathrm{~d}}\end{array}$ & $\begin{array}{c}\text { Corresponding } \\
\text { Probability }\end{array}$ \\
\hline Potential & $G_{\mathrm{av}}$ & 0.919 & $99 \%$ \\
NLFF with preprocessing & $G_{\mathrm{av}}$ & -0.5 & $91 \%$ \\
NLFF with preprocessing & $C_{n}$ & 0.427 & $85 \%$ \\
\hline
\end{tabular}

Table 6. Quicklook IVM subset: Pearson correlation coefficients between $D_{2 \mathrm{~d}}$ and $D_{3 \mathrm{~d}}$ for the potential and the NLFF cases.

\begin{tabular}{cccc}
\hline \hline $\begin{array}{c}\text { Extrapolation } \\
\text { Method }\end{array}$ & $\begin{array}{c}\text { Used 3d } \\
\text { criterion }\end{array}$ & $\begin{array}{c}\text { Correlation } \\
\text { with } D_{2 \mathrm{~d}}\end{array}$ & $\begin{array}{c}\text { Corresponding } \\
\text { Probability }\end{array}$ \\
\hline Potential & $G_{\mathrm{av}}$ & 0.765 & $93 \%$ \\
NLFF with preprocessing & $G_{\mathrm{av}}$ & 0.238 & $65 \%$ \\
NLFF with preprocessing & $C_{n}$ & 0.099 & $30 \%$ \\
\hline
\end{tabular}

Table 7. Pearson correlation coefficients of the fractal dimensions $D_{2 \mathrm{~d}}$ between the unpreprocessed and the preprocessed photospheric magnetograms in the distinct data subsets.

\begin{tabular}{cccc}
\hline \hline & $\begin{array}{c}\text { Data } \\
\text { Subset }\end{array}$ & $\begin{array}{c}\text { Correlation } \\
\text { Coefficient }\end{array}$ & $\begin{array}{c}\text { Corresponding } \\
\text { Probability }\end{array}$ \\
\hline Fully Inverted IVM & Pearson & 0.770 & $99 \%$ \\
HINODE & Pearson & 0.989 & $99 \%$ \\
Quicklook IVM & Pearson & 0.688 & $95 \%$ \\
\hline
\end{tabular}

distinct dataset separately. Although substantial differences exist between the various datasets, it is evident that the results found for the total dataset are qualitatively retained also for the subsets of data coming from different instruments. The correlation between $D_{2 \mathrm{~d}}$ and $D_{3 \mathrm{~d}}$ is significant for potential extrapolation, whereas it is absent in the NLFF limit. Preprocessing does not significantly alter the photospheric magnetic fields in any case.

\section{Discussion and conclusions}

This study investigates whether the complexity of the photospheric magnetic field correlates with the complexity of the UnVos formed in the corona. Using 38 vector magnetograms, we

- estimate the fractal dimension $D_{2 \mathrm{~d}}$ from the pixels with a magnetic field strength exceeding a given threshold $B_{\mathrm{cr}}$ at the photospheric level through a standard box-counting method;

- extrapolate the magnetic field from the photospheric boundary using a

1. nonlinear force-free optimization algorithm with preprocessing at the photospheric level;

2. standard potential extrapolation algorithm;

- calculate $D_{3 \mathrm{~d}}$ by box-counting the sites within our cubic grid, which exceeds a threshold in

1. the averaged magnetic field gradient $G_{\mathrm{av}}$ with respect to their neighboring sites;

2. the normalized magnetic field curl $C_{\mathrm{n}}$ (only for the NLFF fields);

- investigate whether there is any correlation between the inferred $D_{2 \mathrm{~d}}$ and $D_{3 \mathrm{~d}}$. 
Our results show that there is no correlation revealed between $D_{2 \mathrm{~d}}$ and $D_{3 \mathrm{~d}}$ for the NLFF case. The spatial distribution of the UnVos with height shows that $\geq 80 \%$ of the magnetic discontinuities are accumulated in the lower corona (within $20 \mathrm{Mm}$ from the photosphere). The system is evidently highly unstable at these heights, yielding processes that are clearly nonlinear. It is this strong nonlinearity at lower layers that does not allow the corona to respond proportionally to changes imposed by the photospheric driver or, conversely, does not allow the line-tied photosphere to strongly respond to a restructuring of the coronal magnetic fields caused by an energetic event (flare, CME). The results of Sudol \& Harvey (2005) establish that, even for the largest flares, the photospheric response is significant, but rather small (median value of photospheric magnetic field change at $90 \mathrm{G}$ ) even given that these flares have to occur close to the photosphere where most of the free magnetic energy resides.

In contrast to the results for NLFF fields, we have obtained a significant correlation between $D_{2 \mathrm{~d}}$ and $D_{3 \mathrm{~d}}$ in the case of potential field extrapolations. As already explained, this should be attributed to the absence of currents, which forces the coronal magnetic structures to closely follow the photospheric driver. Therefore, the currents in the NLFF extrapolated fields lead to a more complex magnetic topology and the loss of correlations with the photospheric fields.

It is interesting to notice the strong accumulation of UnVos close to the lower (photospheric) boundary in both the potential and the NLFF fields. We believe this is not an artifact and that it has to do with the fine, fibril structure of the photospheric magnetic fields, which gradually fades as we move toward the corona. The structure of these forced fields is long known (Livingston \& Harvey 1969; Howard \& Stenflo 1972; Stenflo 1973) and it becomes less prominent at higher layers until the magnetic field fills the entire coronal volume, excluding small isolated areas of current sheets and tangential discontinuities in general (Parker 2004, and references therein). UnVos are designed to indicate these discontinuities and, as such, they will strongly accumulate at lower layers. This will happen regardless of the extrapolation method that will tend to create smooth fields in the volume, at the same time accommodating the finely structured lower boundary.

Further indications regarding of the absence of correlation between the photospheric and the coronal processes and structures are provided by Metcalf et al. (1994), who examined the spatial and temporal relationship between coronal structures observed with the Soft X-ray Telescope (SXR) on board Yohkoh spacecraft and the vertical electric current density derived from photospheric vector magnetograms. Metcalf et al. (1994) found no evidence directly linking the electric currents observed in the photosphere to the heating of the coronal plasma indicated by the SXR brightness and temperature.

The work of Aschwanden and Aschwanden (2008a,b) - investigating the relationship between the fractal dimension of flare images captured by TRACE (in 2d) to the fractal dimension of coronal arcades produced by an analytical geometric model (in $3 \mathrm{~d}$ ) - indicates a complex relation between $D_{2 \mathrm{~d}}$ and $D_{3 \mathrm{~d}}$. From our results, even this relation may be destroyed when fewer simplifications are used and the forced photospheric fields are taken into account. That the photospheric fields include significant Lorentz forces has been shown by Metcalf et al. (1995) and Georgoulis \& LaBonte (2004).

Our own and previous independent results seem to support the conjecture that the absence of correlation between the photospheric and coronal fractal dimensions would still be the case (in fact, correlation should probably become worse) if a more realistic static or dynamic non-force-free modeling of the coronal field was used. Photospheric turbulence remains the driver for the coronal instabilities, but the strong nonlinearity of the system in the lower coronal layers destroys any kind of direct relation between the photospheric structures and their coronal counterparts. The photospheric driver forces the system to accumulate a large number of magnetic discontinuities that store enough energy to explain the statistical properties of the solar activity in case of release. These discontinuities form patterns that do not follow the morphological properties of the photospheric magnetic flux concentrations, but have a strong impact on the expected dynamical activity of the system, namely, the magnetic energy release and the subsequent particle acceleration processes (Vlahos et al. 2004).

Acknowledgements. We are grateful to Thomas Wiegelmann for kindly contributing his NLFF extrapolation code to this analysis and to Bruce Lites who provided useful information regarding the Level 1D SOT/SP magnetograms from Hinode. Hinode is a Japanese mission developed and launched by ISAS/JAXA, with NAOJ as domestic partner and NASA and STFC (UK) as international partners. It is operated by these agencies in co-operation with ESA and NSC (Norway). IVM magnetograms were obtained by the staff of the U. of Hawaii Mees Solar Observatory, Air Force Office of Scientific Research contract F49620-03-C-0019. IVM quicklook data were obtained from work supported by the National Science Foundation under Grant No. 0454610. Any opinions, findings, and conclusions or recommendations expressed in this material are those of the authors and do not necessarily reflect the views of the National Science Foundation (NSF). Eva Ntormousi and Tassos Fragos are acknowledged for their constructive comments in the course of this work. Finally, we would like to thank the referees for their very constructive comments.

\section{References}

Abramenko, V. I. 2005, Sol. Phys., 228, 29

Aschwanden, M. J., \& Aschwanden, M. J. 2008a, ApJ, 674, 530

Aschwanden, M. J., \& Aschwanden, M. J. 2008b, ApJ, 674, 544

Balke, A. C., Schrijver, C. J., Zwaan, C., \& Tarbell, T. D. 1993, Sol. Phys., 143, 215

Bovelet, B., \& Wiehr, E. 2001, Sol. Phys., 201, 13

Cadavid, A. C., Lawrence, J. K., Ruzmaikin, A. A., \& Kayleng-Knight, A. 1994, ApJ, 429, 391

Conlon, P. A., Gallagher, P. T., McAteer, R. T. J., et al. 2008., Sol. Phys., 248, 297C

Falconer, D. A., Moore, R. L., \& Gary, G. A. 2006, ApJ, 644, 1258

Fragos, T., Rantsiou, E., \& Vlahos, L. 2004, A\&A, 420, 719

Gallagher, P. T., Phillips, K. J. H., Harra-Murnion, L. K., \& Keenan, F. P. 1998, A\&A, 335, 733

Georgoulis, M. K. 2005, ApJ, 629, 69

Georgoulis, M. K. 2008, Geophys. Res. Lett., 35, L06S02

Georgoulis, M. K., \& LaBonte, B. J. 2004, ApJ, 615, 1029

Georgoulis, M. K., Rust, D. M., Bernasconi, P. N., \& Schmieder, B. 2002, ApJ, 575,506

Harvey, K. L., \& Zwaan, C. 1993, Sol. Phys., 148, 85

Hewett, R. J., Gallagher, P. T., McAteer, R. T. J., et al. 2008., Sol. Phys., 248, $311 \mathrm{C}$

Hirzberger, J., Vazquez, M., Bonet, A., Hanslmeier, A., \& Sootka, M. 1997, ApJ, 480, 406

Howard, R., \& Stenflo, J. O., 1972, Sol. Phys., 22, 402

Janssen, K., Vogler, A., \& Kneer, F. 2003, A\&A, 409, 1127

Isliker, H. 1992, Phys. Lett. A, 169, 313

Isliker, H., Anastasiadis, A., \& Vlahos, L. 2000, A\&A, 363, 1134

LaBonte, B. J., Mickey, D. L., \& Leka, K. D. 1999, Sol. Phys., 189, 1

Lawrence, J. K. 1991, Sol. Phys., 135, 249

Lawrence, J. K., \& Schrijver, C. J. 1993, ApJ, 411, 402

Lawrence, J. K., Cadavid, A. C., \& Ruzmaikin, A. A. 1996, ApJ, 465, 425

Lites, B. W., Elmore, D. F., \& Streander, K. V. 2001, in Advanced Solar Polarimetry - Theory, Observation, and Istrumentation, ed.: M. Sigwarth, ASP Conf. Ser., 236, 33

Livingston, W., \& Harvey, J., 1969, Sol. Phys., 10, 294

McAteer, R. T. J., Gallagher, P. T., \& Ireland, J. 2005, ApJ, 631, 628

Metcalf, T. R., Canfield, R. C., Hudson, H. S., Mickey, D. L., \& Wulser, J. P. 1994, ApJ, 428, 860

Metcalf, T. R., Jiao, L., McClymont, A. L. \& Canfield, R. C. 1995, ApJ, 439, 474 
Metcalf, T. R., Derosa, M. L., Schrijver, C. J., et al. 2008, Sol. Phys., 247, 269 Meunier, N. 1999, ApJ, 515, 801

Meunier, N. 2004, A\&A, 420, 333

Mickey, D. L., Canfield, R. C., LaBonte, B. J., et al. 1996, Sol. Phys., 168, 229

Parker, E. N. 2004, Phys. Plasmas, 11, 2328

Priest, E. R., Hornig, G., \& Pontin, D. I. 2003, J. Geophys. Res. Space Phys., 108,1285

Regnier, S., \& Priest, E. R. 2007, ApJ, 669, 53

Roudier, T., \& Muller, R. 1987, Sol. Phys., 107, 11

Schrijver, C. J. 2007, ApJ, 655, 117

Schrijver, C. J., Zwaan, C., Balke, A. C., Tarbell, T. D., \& Lawrence, J. K., 1992, A\&A, 253, L1

Schrijver, C. J., Derosa, M. L., Metcalf, T. R., et al. 2006, Sol. Phys., 235, 161

Seiden, P. E., \& Wentzel D, G. 1996, ApJ, 460, 522
Shimizu, T. 2004, Sol. Phys., 325, 3S

Stark, B., Adams, M., Hathaway, D. H., \& Hagyard, M. J. 1997, Sol. Phys., 174, 297

Stenflo, J. O. 1973, Sol. Phys., 32, 41

Sudol, J. J., \& Harvey, J. W. 2005, ApJ, 635, 647

Tarbell, T., Acton, D., Topka, K., et al. 1990, BAAS, 22, 878

Vlahos, L., \& Georgoulis, M. K. 2004, ApJ, 603, 61

Vlahos, L., Fragos, T., Isliker H., \& Georgoulis M. K. 2002, ApJ, 575, 87

Vlahos, L., Isliker H., \& Lepreti, F. K. 2004, ApJ, 608, 540

Wentzel, D. G., \& Seiden, P. E. 1992, ApJ, 390, 280

Wheatland, M. S., Staurrock, P. A., \& Roumeliotis, G. 2000, ApJ, 540, 1150

Wiegelmann, T. 2004, Sol. Phys., 219, 87

Wiegelmann, T. 2008, J. Geophys. Res., 113, A03S02

Wiegelmann, T., Inhester, B., \& Sakurai, T. 2006, Sol. Phys., 233, 215 